\title{
Organic Chemistry at Chemically Resistant Polymer Surfaces: Modification of Surface Reactivity and Surface Properties
}

\begin{abstract}
Thomas J. McCarthy*
Abstract. This contribution will describe strategies for carrying out surface-selective modification reactions of fluoropolymers at the solution-polymer surface interface. The effects of solvent, temperature, and reagent structure on reactivity and surface selectivity will be discussed. The objective of these modification reactions is to introduce versatile organic functional groups to the surfaces of chemically resistant polymer surfaces; chemical transformations of the functional groups can then be carried out without affecting the structure of the inert 'support' film. Surface properties of these film samples can, thus, be correlated with surface-chemical structure. The chemical reactivity of the surface functional groups, particularly the $\mathrm{OH}$ group, will be discussed in some detail. The thermal stability and thermal reconstruction path of one modified surface will be described; the correlation of coefficient of friction with surface structure will be discussed. The synthesis of a surface that was designed to spontaneously adhere to metal oxides will also be presented.
\end{abstract}

\section{Introduction}

Chemistry at polymer surfaces and interfaces impacts on the majority of polymer materials applications. We have been carrying out a research program directed at preparing specific polymer surfaces with well-defined chemical compositions. With these model substrates, we are attempting to draw surface-structure property and structure-reactivity relations and to predict particular properties with knowledge of the chemical structure. A complementary objective is to impart desired surface properties by introducing specific functional groups in specific locations and densities to polymer film substrates. We have chosen to study chemically resistant (unreactive) polymers containing versatile organic functional groups at their surfaces to meet these objectives. The advantages of these materials as substrates have been described [1]. The most important one is that polymer film samples with inert bulks and reactive surfaces can be modified using a range of conditions which restrict changes to surface chemical ones and do not alter the structure or properties of the bulk. Surface property changes upon reaction can, thus, be ascribed to surface chemical changes.

\section{Preparation of Reactive Surfaces}

A problem inherent to introducing a thin layer of functional groups to the surface of a chemically resistant polymer film is the lack of reactivity of the film substrate; corrosion and pitting due to autocatalysis tend to occur giving rise to surfaces not appropriate for property studies. Different strategies are used to surface-selectively functionalize poly(chlorotrifluoroethylene) (PCTFE) [1], poly(tetrafluoroethylene) (PTFE) [2], poly(vinylidene fluoride) $\left(\mathrm{PVF}_{2}\right)$ [3], and poly(tetrafluoroethylene-co-hexafluoropropylene) (FEP) [4]. In general, a heterogeneous reaction is carried out at the interface between the film substrate and a solvent containing a reagent specific for the solid polymer. A sharp (not diffuse) interface between the film sample and the reactive solution is designed through choice of solvent and reaction temperature.

Each polymer surface modification is a complex process, and there is a great deal of latitude in processes system-to-system. Several factors control the surface selectivity of the reaction and the ultimate interface structure of the product:

1) The Unreacted Polymer-Solvent Interface. A solid polymer in contact with a solvent may interact with the solvent to varying extents along a continuum ranging from not being wet by the solvent to (ultimately) being dissolved by the solvent. The interface can, thus, vary from sharp to diffuse, and because solvent facilitates reaction, the depth (into the solid polymer) of the modification reaction, and, therefore, the thickness of the modified layer in the product will be affected by the nature of this interface.

2) The Product Polymer-Solvent Interface. As a reaction proceeds at a solid polymer-solvent interface, the structure of the interface changes. The product (modified polymer surface) can interact with the solvent to a greater or lesser extent than does the unreacted polymer surface. This can lead to a continuum of products ranging from a thin modified layer to a modified polymer which is soluble.

3) Reagent Solubility. The solubility of the reagent(s) in the unreacted polymer, the modified polymer, the interphase region (wet or swollen unreacted and modified polymer) - which changes throughout the reaction, and solvent have to be considered. The same factors which have been discussed for solvents are equally important for reagents. The reagent will partition among the regions of the system and the dynamic partition coefficients (changing throughout the reaction) will affect product structure.

4) Interface Chemistry. In addition to how the modification reaction affects the solvent and reagent affinities of the solid polymer, the chemistry can effect other properties. If the solution interacts strongly with the product, but cross-linking occurs in the modification reaction, the product will not dissolve, and a thick modified layer will result. If the modification cleaves polymer chains, low-molecularweight material will ablate, and chain ends will be important chemical features.

5) Isolated Product Structure. The structure of the modified solid polymer in contact with the reaction solution may vary significantly from the structure of the isolated product which is free of solvent and reagents. The compatibility (or lack of compatibility) of the unreacted and modified polymer and the disparity between surface free energies may induce reorganizations during rinsing procedures and solvent removal that segregate components and concentrate either modified or unreacted polymer at the surface.

$\mathrm{PVF}_{2}$ is modified by reaction with bases [3] [5] [6]. When $\mathrm{PVF}_{2}$ is immersed in aqueous $\mathrm{NaOH}$ at room temperature, there is no reaction, but when a small amount of $\mathrm{Bu}_{4} \mathrm{~N}^{+}$ion is included in the solution, a rapid dehydrofluorination occurs. A sharp interface exists between the polymer and the solution (there is no "wetting'), and hydroxide (in the absence of tetrabutylammonium) in solution cannot access the surface. $\mathrm{Bu}_{4} \mathrm{~N}^{+}$serves as a phasetransfer catalyst or 'wetting agent' and transports hydroxide to the interface where it effects the dehydrofluorination. The modified polymer does not interact with the solution (there is no 'swelling')

\footnotetext{
"Correspondence: Prof. Dr. T. J. McCarthy Polymer Science and Engineering Department University of Massachusetts

Amherst, Massachusetts 01003, USA
} 
and when the accessible polymer repeat units have been dehydrofluorinated, the reaction stops; we refer to this as 'autoinhibition'. $\mathrm{PVF}_{2}-\mathrm{CH}=\mathrm{CF}-$ prepared under these conditions has a uniform surface layer of less than $10 \AA$ thickness. Increasing the reaction temperature influences the outcome: at $40^{\circ}$, an autoinhibitive reaction occurs to a depth of $\sim 40 \AA$; at $80^{\circ}$ a deeper reaction, which does not display autoinhibition, proceeds giving a more diffuse $\mathrm{PVF}_{2}-\mathrm{PVF}_{2}-\mathrm{CH}=\mathrm{CF}$-interface, Dehydrofluorination of $\mathrm{PVF}_{2}$ with other bases in organic solvents proceeds very differently. Using 1,8-diazabicyclo[5.4.0]undec7 -ene (DBU) in heptane produces a deeply (this depends on reaction temperature) modified surface, but significant amounts of unreacted $\mathrm{PVF}_{2}$ remain in the outer $40 \AA$ (as assessed by XPS), thus the $\mathrm{PVF}_{2}$ $\mathrm{PVF}_{2}-\mathrm{CH}=\mathrm{CF}-$ interface is diffuse (rough).

PTFE and FEP are modified with single-electron reducing agents [2] [4]; we have studied sodium naphthalide and dipotassium benzoin dianion. The products, which we abbreviate PTFE-C and FEP-C, are oxidation-sensitive complex carbonaceous materials. We do not understand the reaction well in terms of the relative importance of diffusion of reducing agent into the product and reduction by electrons transported through the electronically conducting product to virgin polymer; at room temperature and above the reaction with sodium naphthelide is rapid and corrosive yielding very diffuse interfaces. At lower temperatures, the reduction of FEP film with sodium naphthalide can be controlled. At $-78^{\circ}$, the modified layer thickness can be controlled quite accurately with reaction time in the range of $45-90 \AA$; the FEP/FEP-C interface is sharp as evidenced by a combination of XPS, VIS/UV and gravimetric analysis.

PCTFE is modified by reaction with lithium reagents [1] [7]. Alkyllithium reacts by metal-halogen exchange to yield alkyl chloride and lithiated polymer which, in turn, eliminates $\mathrm{LiF}$ to form a difluoroolefin. A second equivalent of alkyllithium adds to the difluoroolefin and $\mathrm{LiF}$ is eliminated, yielding a fluoroolefin containing the alkyl group. Both the depth of reaction into PCTFE and the diffuseness of the PCTFE/PCTFE- $R$ interface are dependent on the reaction temperature, reaction time, and solvent, and very dependent on the structure of the alkyllithium. The degree to which the reaction solvent interacts with the modified product is the major controlling factor. We have prepared modified layers ranging in thickness from $\sim 10 \AA$ (certain conditions show autoinhibition) to thousands of angstroms.

\section{Reactions of Functionalized Surfaces}

Hydroxy groups have been introduced to each of the surfaces described above. In each case, the $\mathrm{OH}$ group reacts as a nucleophile with reagents in solution in contact with the surface; yields are often low. We ascribe the poor yields to surface-steric congestion. PCTFE-OH contains alcohols attached to the polymer by $3-C$ spacers and yields of reactions of this surface are high. This surface is prepared by reaction of PCTFE film with the protected alcoholcontaining $\mathrm{Li}$ reagent, acetaldehyde lithiopropyl ethyl acetal, and subsequent deprotection. The density of $\mathrm{OH}$ groups (the depth of the modification reaction) in the modified film surface (PCTFE-OH) can be controlled with reaction temperature. PCTFE-OH has been converted to a range of modified surfaces using standard $\mathrm{OH}$ group transformations: reaction with $\mathrm{TsCl}$ yields the $p$-toluenesulfonate (PCTFEOTs). The tosylate can be displaced by nucleophiles. PCTFE-OH reacts with acid chlorides to yield the corresponding surface-confined esters and isocyanates to yield urethanes. Chlorosilanes react with PCTFE-OH to yield (surface alkoxy)silanes. $\mathrm{SOCl}_{2}$ reacts with $\mathrm{PCTFE}-\mathrm{OH}$ to give the sulfite.

\section{Thermal Reconstruction of \\ Surface-Functionalized Poly(chlorotrifluoroethylene) [8]}

Modified PCTFE surfaces which were appropriate for following thermal reconstruction by XPS were prepared as outlined above. 2-(Lithiomethyl)-4,4dimethyloxazoline reacts to incorporate trimethyloxazoline functionality in the outer $10-20 \AA$ of the PCTFE film (PCTFE-TMO). Hydrolysis of PCTFETMO renders surface carboxylic-acid groups (PCTFE-COOH). Hydroxyl groups are incorporated by reaction of PCTFE with 3-lithiopropyl ethyl acetal and subsequent hydrolysis (PCTFE-OH). The thickness of the modified layer in PCTFE-OH depends on the conditions of reaction; we have studied film samples with modified layers of $\sim 30-\AA$ and $\sim 1150-\AA$ thickness. PCTFE-OH $(30 \AA)$ was further modified to yield PCTFE$\mathrm{OC}(\mathrm{O}) \mathrm{CH}_{3}$, PCTFE-OC(O) $\mathrm{CF}_{3}$, and PCTFE-OSiMe ${ }_{3}$. All of the surfaces are stable (give reproducible surface analytical data) indefinitely at room temperature. On heating for days at $80^{\circ}-110^{\circ}$ the surface regions of PCTFE-TMO, PCTFE-OH (30 $\AA)$, PCTFE-OH (1150 $\AA$ ), PCTFE$\mathrm{COOH}$, and PCTFE-OC(O) $\mathrm{CH}_{3}$ reconstruct as evidenced by contact angle analysis. XPS indicates the reconstruction of PCTFE-TMO and PCTFE-OH (30 $\AA$ ), but little or no changes in XPS spectra occur on heating PCTFE-OH $(1150 \AA)$, PCTFE-COOH and PCTFE-OC(O) $\mathrm{CH}_{3}$. PCTFE-OC(O)CF ${ }_{3}$ and PCTFE-OSiMe show no tendency to reconstruct. A reconstruction process which involves the concomitant migration of modified repeat units from the outer $10 \AA$ of the film sam- ple to a deeper region and migration of unmodified PCTFE repeat units from a deeper region into the outermost $10 \AA$ is evident in PCTFE-TMO and PCTFE-OH $(30 \AA)$. The distance scale of these motions is small; the majority of the modified repeat units remains in the outer $10 \AA$. Another reorganization process is evident from contact angle measurements of PCTFE-OC(O)CH $\mathrm{CH}_{3}$ and PCTFE-OH $(1150 \AA)$ and it involves motions of even smaller distance; repeat units do not measurably migrate (the composition of the outermost $10 \AA$ remains constant), and we propose that they rotate.

\section{Surface Chemistry: Coefficient of Friction Correlations [9]}

The frictional properties of polychlorotrifluoroethylene (PCTFE) film and surface-modified PCTFE films sliding on polyethylene terephthalate (PET) film have been studied. Modified surfaces studied, thus far, are an acetal (the protected alcohol surface described above), a hydroxyl (PCTFE-OH), and the acetate, butyrate, decanoate, stearate and heptafluorobutyrate esters (PCTFE-OAc, PCTFEOBut, PCTFE-ODec, PCTFE-OSt PCTFE-OHFB). The coefficient of sliding friction, $\mu$, was measured using a homebuilt instrument. Wear effects were analyzed by scanning electron microscopy (SEM), X-ray photoelectron spectroscopy (XPS), and water-contact angle. Results for thinly $(\sim 30 \AA)$ modified surfaces show no significant changes in $\mu$ for any of the surfaces except the heptafluorobutyrate, where an increase in friction is observed.

\section{Synthesis of a Polymer Surface Containing Covalently Attached Triethoxysilane Functionality: Adhesion to Glass [10]}

The reaction of PCTFE-OH with (3-isocyanatopropyl)triethoxy-silane in the presence of $\mathrm{Bu}_{2} \mathrm{Sn}$ dilaurate produces a polymer surface containing (alkyl)(triethoxy)silane functionality PCTFE-OC(O) NH( $\left(\mathrm{CH}_{2}\right)_{3} \mathrm{Si}(\mathrm{OEt})_{3}$. ATR IR, and XPS results indicate that the reaction proceeds in essentially quantitative yield, and that the (EtO) $)_{3}$ Si moiety is intact and covalently attached to the polymer film surface via a urethane linkage. Acid-catalyzed hydrolysis of PCTFE-OC(O)NH( $\left(\mathrm{CH}_{2}\right)_{3} \mathrm{Si}(\mathrm{OEt})_{3}$ yields a surface containing both siloxane and silanol functionality: PCTFE-OC(O) $\mathrm{NH}\left(\mathrm{CH}_{2}\right)_{3} \mathrm{Si}(\mathrm{O}) \mathrm{OH}$. PCTFE-OC(O)$\mathrm{NH}\left(\mathrm{CH}_{2}\right)_{3} \mathrm{Si}(\mathrm{OEt})_{3}$ adheres tenaciously to glass slides: when placed in contact under mild heat $\left(80^{\circ}\right)$ and pressure, the polymer film (5 mil) cannot be removed from the glass without tearing the film. SEM and XPS analyses of the glass surface indicate that cohesive failure in PCTFE occurs on 
delamination. PCTFE, PCTFE-OH and PCTFE-OC(O)NH( $\left.\mathrm{CH}_{2}\right)_{3} \mathrm{Si}(\mathrm{O}) \mathrm{OH}$ films do not adhere to glass under the same conditions.

I thank the co-authors listed in the references.
[1] A.J. Dias, T. J. McCarthy, Macromolecules 1987 $20,2068$.

[2] C.A. Costello, T.J. McCarthy, Macromolecules 1987, 20, 2819.

[3] A.J. Dias, T. J. McCarthy, Macromolecules 1984 $17,2529$.

[4] R.C. Bening, T.J. McCarthy, Macromolecules 1990, 23, 2648

[5] J. V. Brennan, T. J. McCarthy, Polym. Prep. (Am Chem. Soc., Div. Polym. Chem.) 1989, 30, 152.
[6] J.V. Brennan, T.J. McCarthy, Polym. Prepr. (Am. Chem. Soc., Div. Polym. Chem.) 1987, 29, 338

[7] K.-W. Lee, T.J. McCarthy, Macromolecules 1988 21, 2318.

[8] E.M. Cross, T.J. MeCarthy, Macromolecules, in press.

[9] T.G. Bee, T.J. McCarthy, work in progress.

[10] K.-W. Lee, T.J. McCarthy, Macromolecules 1988 21,3353 .

\title{
Adhesive Bonding: The Importance of Polymeric Interfaces
}

\author{
Anthony J. Kinloch*
}

\section{Introduction}

Modern adhesives are increasingly being used in advanced technology applications and are based upon macromolecules. Thus, all interfaces between adhesives and substrates may be said to constitute a 'polymeric interface'. In the formation of an adhesive joint three important stages may be identified [1]: Firstly, the adhesive must establish intimate interfacial molecular contact with the substrate - often termed 'wetting'. Some form of intrinsic adhesion forces then need to be established between the adhesive and the substrate, and such intrinsic adhesion forces will hold the materials together throughout the service-life of the adhesive joint. Secondly, to attain the above requirements, the adhesive had to be in a 'liquid' form at some stage during the bonding operation. However, the adhesive almost invariably now has to harden in order to be able to withstand the stresses and strains that may be applied to the joint. Thirdly, it must be appreciated that the details of the design of the joint, the way in which loads are applied to it, and the service environment that it must withstand, will all affect its mechanical performance and life expectancy.

The importance of the polymeric interface is to be found throughout all these different aspects of the science of adhesion and adhesives, and this will be illustrated by examples drawn from the bonding of

\footnotetext{
* Correspondence: Dr. A.J. Kinloch

Imperial College of Science, Technology and Medicine Department of Mechanical Engineering Exhibition Rd.

London, SW7 2 BX, UK
}

elastomers to plastic substrates, the bonding of thermoplastic composites, and the environmental attack of moisture upon steel and aluminium-alloy bonded joints.

\section{The Bonding of Elastomers to Plastic Substrates}

The thermodynamic work of adhesion, $W_{\mathrm{A}}$, required to separate a unit area of a solid and a liquid phase forming an interface across which secondary forces are acting may be related to the surface and interfacial free energies by the Dupre equation:

$W_{\mathrm{A}}=\gamma_{\mathrm{a}}+\gamma_{\mathrm{s}}-\gamma_{\mathrm{as}}$

where $\gamma_{\mathrm{a}}$ is the surface free energy of the adhesive, $\gamma_{\mathrm{s}}$ that of the substrate, and $\gamma_{\mathrm{a}} \mathrm{s}$ that of the adhesive/substrate interface.

Now by adopting a fracture mechanics approach the work of Andrews, Gent and Kinloch [2-5] defined a geometry-independent measure of joint strength, the adhesive fracture energy, $G_{c}$. From experimental and theoretical considerations, it was demonstrated that $G_{\mathrm{c}}$, for a cross-linked elastomeric adhesive/rigid plastic interface could be divided into two major components: a) The energy required to propagate a crack through a unit area of interface in the absence of viscoelastic energy losses, i.e. an intrinsic adhesive fracture energy, $G_{0}$, which is a direct measure of the bonding forces. b) The energy, $\psi$ dissipated viscoelastically within the elastomeric adhesive at the propagating crack, again referred to unit area of interface.

The values of $G_{\mathrm{o}}$ were ascertained for various joints from studies of the crack propagating along the elastomeric/substrate interface. When interfacial failure occurred, the values of $G_{\mathrm{o}}$ were compared to corresponding values of $W_{A}$ calculated from Eqn. 1 . The results are shown in Table $I$ and, as may be seen, the values of the two parameters are in good agreement. Thus, the nature of the intrinsic adhesion forces acting across these interfaces was secondary interfacial bonds.

An important general point emerges from the above work. Namely, that although the energy, $\psi$, which is dissipated viscoelastically and plastically is usually far greater than $W_{\Lambda}$, the value $\psi$ is dependent upon the value of $W_{A}$. Indeed, for the model joints described above, the value of $\psi$ is directly proportional to the value of $W_{\mathrm{A}}$. Hence, designing to maximise the interfacial bonding forces is not a trivial pursuit. Obviously, this has long been recognised in industry as evidenced by most adhesives being polar in character and often having the capability to establish $\mathrm{H}$-bonds, and possibly even chemical bonds, to the substrate.

Table 1. Values of the Intrinsic Fracture Energy $\left(G_{\mathrm{o}}\right)$ and the Thermodynamic Work of Adhesion. $\left(W_{\mathrm{A}}\right)$ for a Cross-linked Styrene-Butadiene Rubber Adhering to Varios Substrates

\begin{tabular}{lll}
\hline Substrate & $G_{\mathrm{O}}\left[\mathrm{mJ} / \mathrm{m}^{2}\right]$ & $W_{\mathrm{A}}\left[\mathrm{mJ} / \mathrm{m}^{2}\right]$ \\
\hline Fluorinated ethylene-prophylene copolymer & 22 & 28 \\
Plasma-treated ethylene-prophylene copolymer & 69 & 57 \\
Poly(chlorotrifluoroethylene) & 75 & 63 \\
Nylon 11 & 71 & 71 \\
Poly(ethylene terephthalate) & 79 & 72 \\
\hline
\end{tabular}

